\title{
Low energy shape oscillations of negative parity in the main and shape-isomeric minima in actinides
}

\author{
M. Kowal* and J. Skalski \\ Soltan Institute for Nuclear Studies, \\ Hoża 69, PL-00-681 Warsaw, Poland
}

\begin{abstract}
We study low energy shape oscillations of negative parity in the first and second (isomeric) minima in actinides. As a main tool we use the phenomenological Woods-Saxon potential with a variety of shape deformations. This allows to include a mixing of various multipolarities when considering oscillations with a fixed $K$ quantum number. The phonon energies are determined either from the collective Hamiltonian with the microscopic-macrocopic energy and cranking mass parameters, or from its simplified version with the constant mass parameters. The results for $K^{\pi}=$ $0^{-}, 1^{-}$in the first minima are in a reasonable agreement with experimental data, including predicted E1 transitions; the $K^{\pi}=2^{-}$energies are systematically overestimated. In the second minimum, as compared to the data for ${ }^{240} \mathrm{Pu}$ and ${ }^{236} \mathrm{U}$, our calculated $K=1,2$ energies are overestimated while the $K=0$ energies are three or more times too large. This signals either a non-collective character of the experimentally assigned $K=0$ states or a serious flaw of the model in the second minimum. More data on the $K=0, I^{\pi}=1^{-}$collective states in the second minima of other nuclei are necessary to resolve this issue.
\end{abstract}

PACS numbers: 21.60.Ev,21.10.Re,21.10.Ky

*Electronic address: mickowal@fuw.edu.pl 


\section{INTRODUCTION}

Recently, a considerable amount of data has been gathered on the nuclear states in the second well in the actinide region, especially in ${ }^{240} \mathrm{Pu}$, see [1] and references cited therein. Various rotational bands, most of them of negative parity, have been identified in this nucleus with moments of inertia characteristic of superdeformation (SD). Such spectroscopic data provide a much wanted test for nuclear models that were originally fitted in a region of deformations around the ground state (g.s.) minima: How much of their predictive power is left beyond that region? Negative-parity shape oscillations are natural candidates for the observed low energy band-heads at the SD. Here we study them within the much used microscopic-macroscopic model based on the deformed Woods-Saxon potential. The found properties of excitations in the shape-isomeric minima provide both a prediction and a test of the model. We check the used method by first applying it to the negative-parity excitations in the first minima in actinides, on which data are more abundant.

Discovery of fast fissioning states in actinides by Polikanov et al. [2] was interpreted soon afterwards by Flerov and Druin [3] and Strutinsky [4] as evidence for very deformed secondary minima in these nuclei. Later experiments provided support for this guess, with measurements of rotational band in ${ }^{240} \mathrm{Pu}[5]$ and then, of the quadrupole moment in the second well in ${ }^{239} \mathrm{Pu}$ [6]. However, very short lifetimes, in the range from 10 ps to 10 ms, and experimental difficulties precluded, and in fact preclude to this very day, gainining sufficient knowledge on the nuclear structure in the second minimum. Only four values of the quadrupole moment in the second well were measured via nuclear methods of the total of 34 shape-isomeric states. Three additional data for Am isotopes come from the optical isotope shift and hyperfine structure measurements of the difference between the mean square radii in the ground and isomeric states [7]. Moreover, difficulties in the experimental access to fission isomers make the results very dependable on rather extended argumentation chains involving assumptions instead of established experimental facts. Although this does not necessarily invalidates the claimed results, quite a number of legitimate reservations may be formulated which diminish their firmness, see [8].

We determine properties of low-lying negative-parity shape vibrations in even-even actinides using the collective model, i.e. the Schrödinger equation with deformation parameters as coordinates. As the ingredients we take the microscopic-macroscopic energy and cranking 
mass parameters. In order to describe nonaxial modes we include nonaxial deformations of the nuclear shape corresponding to $K=1,2,3$. The use of the microscopic-macroscopic energy is consistent with the working assumption that it correctly predicts the shape dependence of energy for "cold" nuclear configurations. On the other hand, using adiabatic mass parameters is only an approximation. It should work reasonably well for phonon energies smaller than $2 \Delta$, with $\Delta$ the neutron or proton pairing gap, The experimental energies in the second well and many in the first well fulfil this condition [1].

One might expect that negative-parity shape vibrations are mostly octupole. However, there are at least two reasons to consider admixtures of higher order odd-multipolarity modes, i.e. $\lambda=5$ and 7: 1) The equilibrium shapes are spheroidal, hence the multipole components of different $\lambda$ are not the normal modes (not even being orthogonal) and may couple with each other, 2) Deformation parameters $\beta_{\lambda \mu}$ of the microscopic-macroscopic model define the s.p. potential, not the density. Therefore, the octupole part of the latter may be produced by various odd- $\lambda$ deformations of the potential. As a consequence, one should account for a possible mode coupling when looking for low lying excitations of negative parity. We are not aware of any other study of shape oscillations explicitely including oddrank multipoles of the order higher than three.

One can observe that an alternative approach to the study of octupole vibrations, the schematic Random Phase Approximation [9 11], while free from the adiabaticity assumption that we use for mass parameters, has its own problems, including the necessity of fixing the coupling constants. In order to study the coupling of various multipolarities, one would have many constants to fix.

\section{METHOD}

We use a sigle-particle (s.p.) Hamiltonian with the deformed Woods-Saxon potential defined in terms of the nuclear surface, according to the scheme exposed in [12]. However, the potential used in the present work admits more general nuclear shapes: The only restriction imposed on them is that they have one symmetry plane $y-z$. This leaves one conserved signature quantum number, $s_{x}= \pm i$, being the eigenvalue of the signature operator $\mathcal{S}_{x}=$ $\mathcal{P} \mathcal{R}_{x}^{-1}$, with $\mathcal{P}$ the intrinsic parity and $\mathcal{R}_{x}$ the rotation by $\pi$ about the intrinsic $x$ axis. The

degeneracy of pairs of states with $s_{x}= \pm i$ (the Kramers degeneracy) reduces by half the 
dimension of the s.p. Hamiltonian matrix.

Nuclear shapes compatible with the assumed symmetry are defined by the following equation of the nuclear surface

$$
\begin{aligned}
R(\theta, \varphi) & =c(\{\beta\}) R_{0}\left(1+\sum_{\lambda>1} \beta_{\lambda 0} Y_{\lambda 0}(\theta, \varphi)\right. \\
& +\sum_{\lambda>1, \mu>0, \text { even }} \beta_{\lambda \mu} Y_{\lambda \mu c}(\theta, \varphi) \\
& \left.+\sum_{\lambda>1, \mu>0, \text { odd }} \beta_{\lambda \mu} Y_{\lambda \mu s}(\theta, \varphi)\right),
\end{aligned}
$$

where $c(\{\beta\})$ is the volume-fixing factor. The real-valued spherical harmonics, $Y_{\lambda \mu c}$ with even $\mu>0$ and $Y_{\lambda \mu s}$ with odd $\mu>0$, are defined in terms of the usual ones as: $Y_{\lambda \mu c}=$ $\left(Y_{\lambda \mu}+Y_{\lambda-\mu}\right) / \sqrt{2}$ and $Y_{\lambda \mu s}=-i\left(Y_{\lambda \mu}+Y_{\lambda-\mu}\right) / \sqrt{2}$. In other words, the dependence of the shape on the azimuthal angle $\varphi$ enters through functions $\cos (\mu \varphi)$ with $\mu$ even and $\sin (\mu \varphi)$ with $\mu$ odd.

For the macroscopic part we used the Yukawa plus exponential model [13]. The parameters of both the macroscopic part and the s.p. potential used in the present work, as well as the way the shell- and pairing corrections are calculated, are the same as in a number of previous studies, e.g. in [14].

\section{A. Oscillations around I and II minima}

The second minima in actinides found in this and previous calculations, see e.g. [15, 16], correspond to the axially- and reflection-symmetric shapes. The same holds for the g.s. minima, except for some light thorium, uranium and plutonium isotopes with $N \leq 138$ that have octupole equilibrium deformations and are not included in the present study. So we

are left with nearly parabolic, not too shallow minima. Small oscillations around them with different intrinsic $K$ numbers or parities are nearly independent. Indeed, the amplitude of vibrations cannot be too large, while exactly at the minimum the modes are uncoupled, except for the Coriolis coupling which may be then considered as a perturbation.

The treatment of negative-parity shape oscillations in the collective model is based on the collective Hamiltonian

$$
\hat{H}=-\frac{\hbar^{2}}{2} \frac{1}{\sqrt{\operatorname{det} B}} \sum_{i, j=1}^{3} \frac{\partial}{\partial \beta_{i}}\left(\sqrt{\operatorname{det} B}\left(B^{-1}\right)_{i j} \frac{\partial}{\partial \beta_{j}}\right)+V\left(\beta_{k}\right)
$$


diagonalized within the space of collective wave functions with the scalar product $\left\langle\psi_{1}\right|$ $\left.\psi_{2}\right\rangle=\int d^{3} \beta_{k} \sqrt{\operatorname{det} B} \psi_{1}^{*} \psi_{2}$. Here $V\left(\beta_{k}\right)$ is the microscopic-macroscopic energy and $B_{i j}\left(\beta_{k}\right)$ is the mass tensor with indices corresponding to deformations $\beta_{\lambda K}, \lambda=3,5,7$. In the present work we use cranking mass parameters. Oscillations for each $K=0,1,2,3$ are considered separately. A similar approach was used in [17] to study the $K=0$ octupole state in ${ }^{222}$ Ra. In contrast to that work, where both quadrupole and octupole coordinate were used, we restrict collective variables to reflection-asymmetric deformations.

Since our study is confined to nuclei with sufficiently deep, nearly parabolic minima, we can use the approximation of small oscillation amplitudes. This formalism follows from the one above if we replace $V$ by the quadratic form $(1 / 2) \sum_{i j} C_{i j} \beta_{i} \beta_{j}$, approximately valid around the potential minimum, with the stiffness coefficients $C_{i j}$, and fix the mass parameters at the values calculated at this minimum. Then the Hamiltonian becomes

$$
\hat{H}=-\frac{\hbar^{2}}{2} \sum_{i, j=1}^{3}\left(B^{-1}\right)_{i j} \frac{\partial^{2}}{\partial \beta_{i} \partial \beta_{j}}+\frac{1}{2} \sum_{i, j=1}^{3} C_{i j} \beta_{i} \beta_{j} .
$$

Within this 3D harmonic oscillator model, the study of the $\lambda=3,5,7$ coupling is straightforward. The eigenmodes $\xi_{k}$ are given by a transformation of coordinates

$$
\beta_{i}=\sum_{k=1}^{3}\left(\sum_{j=1}^{3} \sqrt{\frac{\hbar}{B_{D j} \Omega_{D k}}} S_{1 i j} S_{2 j k}\right) \xi_{k}
$$

where the orthogonal matrices $S_{1}$ and $S_{2}$ diagonalize the mass tensor, $S_{1}^{T} B S_{1}=B_{D}$, and the frequency matrix $\Omega, S_{2}^{T} \Omega S_{2}=\Omega_{D}$, with $\Omega^{2}=B_{D}^{-1 / 2} S_{1}^{T} C S_{1} B_{D}^{-1 / 2}$. The square of the oscillation frequency $\hbar \omega_{K}$ corresponding to the oscillation with a given $K=0,1,2,3$ is the smallest solution to the cubic equation $\operatorname{det}\left(C_{i, j}-\left(\hbar \omega_{K}\right)^{2} B_{i j}\right)=0$, with $C_{i j}$ and $B_{i j}$ the stiffness and mass $3 \times 3$ matrices, $i, j=\beta_{\lambda K}$ and $\lambda=3,5,7$.

The calculations of the coupled oscillations with the Hamiltonian (2) were made only for selected cases, as they require a time-consuming calculation of mass parameters, especially for $K \neq 0$. Some technical aspects of these calculations, as well as the evaluation of the cranking mass parameters for the rich deformation set Eq. (1), are shortly described in the Appendix A. As discussed in Sect. III below, the results obtained with deformationdependent mass parameters are not very different from those of the much simpler version with constant mass parameters (3). 


\section{B. Electric dipole transitions}

Reduced probabilities of electromagnetic (EM) transitions between the rotational band built on the one-phonon state and the g.s. band can be calculated assuming the fixed structure of both the phonon and the collective rotor [18]. For an operator $\mathcal{M}$ of the multipolarity $\lambda$ one has

$$
B\left(\lambda ; K_{1}=0, I_{1} \rightarrow K_{2}, I_{2}\right)=\left(2-\delta_{K_{2} 0}\right)\left\langle I_{1} 0 \lambda K_{2} \mid I_{2} K_{2}\right\rangle^{2}\left|\left\langle K_{2}\left|\mathcal{M}\left(\lambda, K_{2}\right)\right| K_{1}=0\right\rangle\right|^{2}(5)
$$

with $\mathcal{M}\left(\lambda, K_{2}\right)$ the intrinsic spherical component. For the negative-parity shape vibrations, the most prominent are dipole transitions, if not hindered by special reasons, as the octupole transitions are usually much weaker. It is especially true for shape isomers where only E0 and E1 transitions are observed [27]. We have $\mathcal{M}(E 1,0)=[3 /(4 \pi)]^{1 / 2} \hat{D}_{z}$ and $\mathcal{M}(E 1,1)=$ $-[3 /(4 \pi)]^{1 / 2}\left(\hat{D}_{x}+i \hat{D}_{y}\right) / \sqrt{2}$, where the dipole moment $\hat{\mathbf{D}}=e\left(N \sum_{p} \mathbf{r}_{p}-Z \sum_{n} \mathbf{r}_{n}\right) / A$. Since the $K=1$ phonon is the equal-weight combination of the equal-energy phonons in directions $x$ and $y$, the intrinsic matrix element in Eq. (5) equals $-[3 /(4 \pi)]^{1 / 2} D_{y}^{t}$, where $D_{y}^{t}=\left\langle Y_{\lambda 1 s}\right|$ $\hat{D}_{y} \mid$ g.s. $\rangle$, with symbolically denoted one-phonon state induced by the deformations $Y_{\lambda 1 s}$, $\lambda=3,5,7$, Eq.(1).

Transition matrix elements in the intrinsic frame between the g.s. and the lowest excited state of negative parity $|\pi-\rangle$ could be calculated by integrating the transition density $\rho_{t r}\left(\beta_{k}\right)=\left\{\sqrt{\operatorname{det} B} \psi_{\pi-}^{*} \psi_{g s}\right\}\left(\beta_{k}\right)$ with the proper operator, represented in the collective space. Taking the dipole moment as an example, we have

$$
D^{t}=\langle\pi-|\hat{D}| 0\rangle=\int_{-\infty}^{\infty} d^{3} \beta_{k} \rho_{t r}\left(\beta_{k}\right) D\left(\beta_{k}\right)
$$

where $D\left(\beta_{k}\right)$ is the expectation value of $\hat{D}$ in the mean field state with the deformations $\beta_{k}$ [17].

A considerably simpler approximation consists in calculating the diagonal matrix element of the transition operator in the mean-field state with the deformations $\beta_{k}^{t r}$ fixed as the most probable by the above transition density:

$$
\beta_{k}^{t r}=\int_{-\infty}^{\infty} d^{3} \beta_{j} \rho_{t r}\left(\beta_{j}\right) \beta_{k}
$$

The dipole and octupole moment operators are to the leading order linear in the oddmultipole deformation parameters $\beta_{\lambda K}$, so indeed, the integration in Eq. (6) replaces $\beta_{\lambda K}$ in 
$D\left(\beta_{3 K}, \beta_{5 K}, \beta_{7 K}, \ldots\right)$ by $\beta_{\lambda K}^{t r}$. A nonlinearity of $D$ as a function of deformations may introduce an error in this approximation. Assuming that the lowest negative-parity mode corresponds to the normal coordinate $\xi_{1}$, the values of $\beta_{k}^{\text {tr }}$ for harmonic vibrations are actually given by Eq.(41) with $\xi_{1}^{t r}=1 / \sqrt{2}, \xi_{2}=\xi_{3}=0$.

This may be contrasted with the strong coupling limit with two octupole minima, at $\pm \beta_{\lambda K}^{e q}$, in which the transition matrix element $D^{t}$ is calculated as the expectation value at this deformation of equilibrium. One can notice that in this case, $\beta_{\lambda K}^{e q}$ is equal to $\beta_{\lambda K}^{\pi-}$, the expectation value of $\beta_{\lambda K}$ in the first excited state of negative parity, nearly degenerate with the g.s., $\beta_{\lambda K}^{\pi-}=2 \int_{0}^{\infty} \sqrt{\operatorname{det} B}\left|\psi_{\pi-}\right|^{2} \beta_{\lambda K} d^{3} \beta_{k}$. For the harmonic lowest-lying phonon one has the relation $\beta_{\lambda K}^{t r}=0.63 \beta_{\lambda K}^{\pi-}$ which follows from Eq. (4) and the relation for the one-dimensional harmonic oscillator: $\xi_{1}^{t r}=(\pi / 8)^{1 / 2} \xi_{1}^{\pi-} \approx 0.63 \xi_{1}^{\pi-}$, as $\xi_{1}^{\pi-}=2 / \sqrt{\pi}$.

Expectation value of the electric dipole moment in the state with deformations $\beta_{\lambda K}$, $K=0,1$, is calculated as a sum of the macroscopic and shell-corection parts, see e.g. [19, 20]. The macroscopic part, derived within the Droplet Model in [21], has to be calculated as in [22], i.e. without assuming small $\beta_{\lambda \mu}$. For the completeness of the presentation, the relevant formulas and parameters are collected in the Appendix B.

\section{Estimate of the Coriolis coupling effect}

Within the rigid rotor-vibration coupling model, there are two modifications of the energy of stationary states with the total angular momentum $I$ relative to the energy of one-phonon states: (i) a shift by $a\left(I(I+1)-K^{2}\right)$, with $a=\hbar^{2} /(2 \mathcal{J}), \mathcal{J}$ the moment of inertia, and (ii) the effect of the Coriolis coupling

$$
\left\langle I M ; K+1\left|\hat{H}_{C o r}\right| I M ; K\right\rangle=-a \sqrt{1+\delta_{K 0}} \sqrt{(I-K)(I+K+1)}\left\langle K+1\left|\hat{J}_{+}\right| K\right\rangle
$$

connecting states differing by $\Delta K=1$. In the spherical limit, the matrix elements of $\hat{J}_{+}$ between substates $K$ and $K+1$ of a collective vibration of the multipolarity $\lambda$ are equal to $\sqrt{(\lambda-K)(\lambda+K+1)}$. The actual matrix elements for octupole phonons in deformed nuclei were found close to the spherical limit in [9]. Here we also include multipoles $\lambda=5$ and 7 , so we estimate the effect of the Coriolis coupling using the spherical value with $\lambda$ consistent with the composition of the lowest-lying phonon, see sect. III.

For a test case of $I=1$ states (the mixing of $K=0$ and $K=1$ ) in ${ }^{240} \mathrm{Pu}$, in the first 
minimmum, we take $\lambda=3$ and with $a=7.156 \mathrm{keV}$ [23] find the coupling $H_{\text {Cor }}=49.6 \mathrm{keV}$; in the second well, with $a=3.33 \mathrm{keV}$ [1], we obtain $H_{C o r}=23.1 \mathrm{keV}$ for $\lambda=3$ and $36.5 \mathrm{keV}$ for $\lambda=5$. As the energy shifts of $I=1$ states due to the coupling (8) are smaller then the coupling itself, especially when the difference in Coriolis-unperturbed energies is much larger than $H_{C o r}$, which is often the case, we do not include them in presented results.

\section{RESULTS AND DISCUSSION}

We begin with the description of energy minima obtained by a minimization in a multidimensional deformation space. A typical plot of energy vs. deformation $\beta_{20}$ after minimization with respect to other axial deformations is shown in Fig. 1 for ${ }^{240} \mathrm{Pu}$. It is normalized by setting the macroscopic energy to zero at the spherical shape. The experimental excitation energy of the superdeformed state, determined by the statistical analysis, equals $E_{I I}^{e x p}=2.25 \mathrm{MeV}$ [1], whereas our calculation gives $E_{I I}^{t h}=2.0 \mathrm{MeV}$. The calculated first and second barrier, $E_{A}^{t h}=6.4 \mathrm{MeV}$ and $E_{B}^{t h}=5.0 \mathrm{MeV}$, are in a satisfactory agreement with the experimental values $E_{A}^{\exp }=5.8 \mathrm{MeV}$ and $E_{B}^{\exp }=5.45$. As mentioned before, mass-asymmetric and nonaxial deformations do not change the first and second minima.

One can also see in Fig. 1 the calculated third minimum. A detailed discussion of the third minima goes beyond the scope of the present paper. Here, we can mention that nonaxial deformations, not included in Fig. 1, modify the energy surface around them.

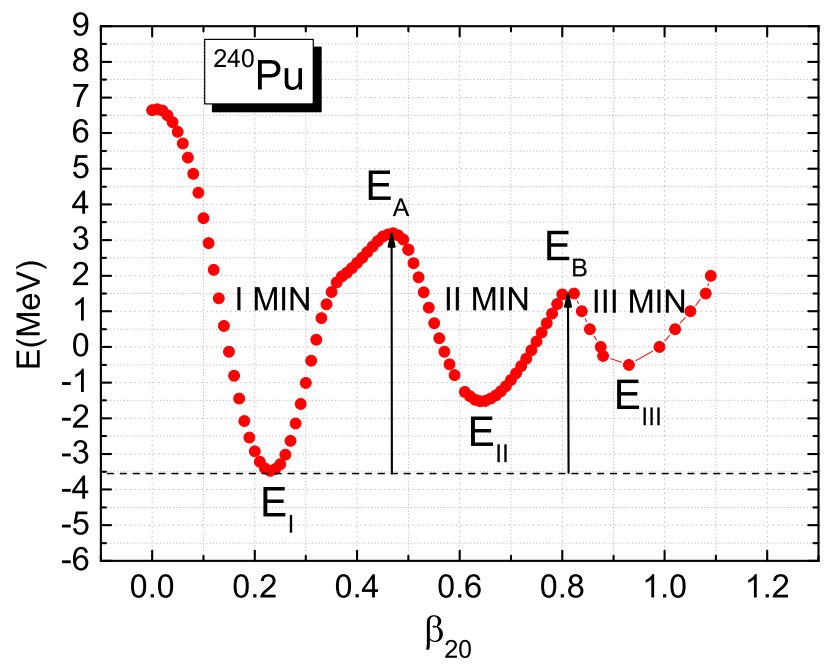

FIG. 1: Calculated fission barrier for ${ }^{240} \mathrm{Pu}$ as a function of $\beta_{20}$. 
Energies of shape vibrations depend on the values of the mass and stiffness parameters in the vicinity of the equilibrium deformation. The stiffness coefficients were determined by fitting energy by a quadratic form in deformations. A quality of this approximation in the first and second well is illustrated in Fig,2 for the octupole deformation $\beta_{30}$. It can be seen that the assumption of harmonicity is satisfied in the reegion of excitation energies up to 2-2.5 MeV. The root-mean-square deviation of the fit for ${ }^{234} \mathrm{U}$ equals $1.3 \mathrm{keV}$ and $0.45 \mathrm{keV}$ at the first and second minimum, respectively. Errors of the fit, for all nuclei and deformations, fall in the range 1-20 keV, with a typical number of points in each fit equal to 30.
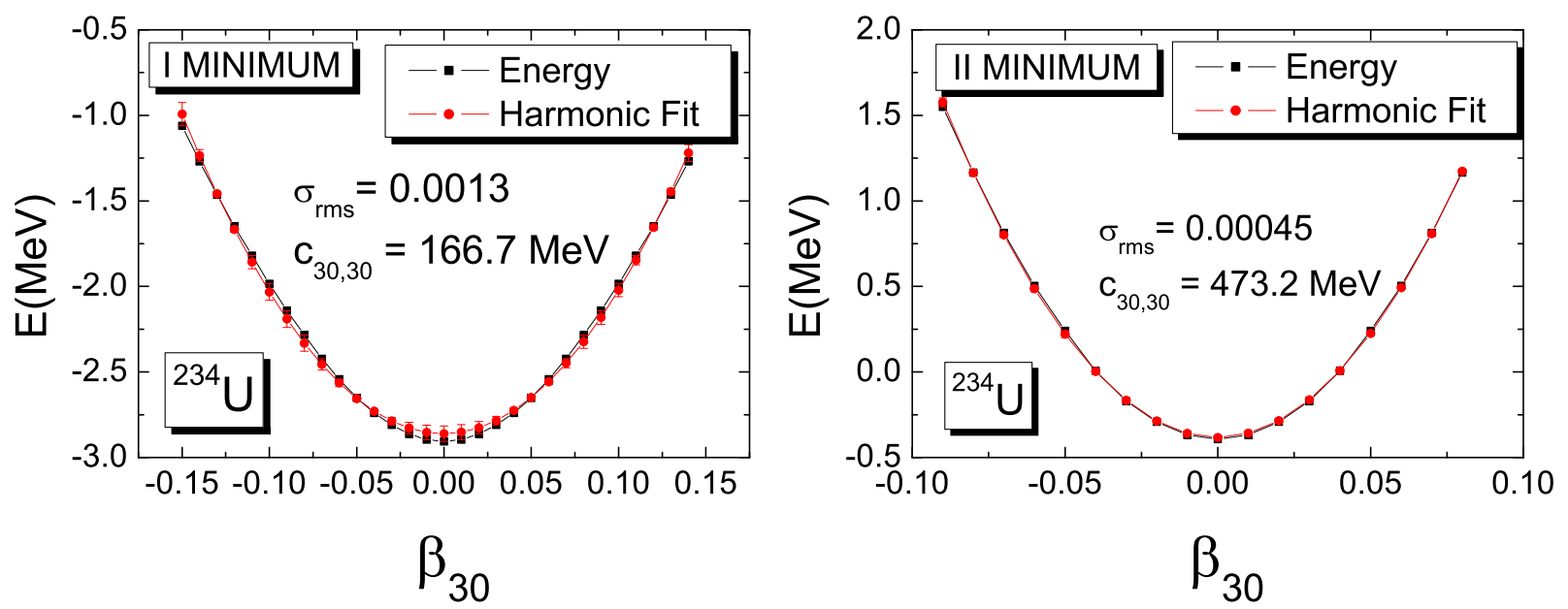

FIG. 2: The extraction of the stiffness $C_{3030}$ at the I-st and II-nd minimum for ${ }^{234} \mathrm{U}$.

A collection of all calculated $K=0$ stiffness coefficients is shown in Fig. 3 for the first minima and in Fig. 4 for the second minima. There are diagonal $C_{3030}, C_{5050}, C_{7070}$ and offdiagonal $C_{3050}, C_{3070}, C_{5070}$ stiffness parameters. It is natural when the latter are smaller than the former. Very telling is the relative smallness of the $C_{3030}$ coefficients at the first minima, where they are similar in magnitude to the off-diagonal coefficients, and their much larger values at the second minima.

The plots of mass parameters $B_{3030}$ and $B_{5050}$ around the reflection- symmetric I and II minimum are shown in Fig. 5. The ${ }^{240} \mathrm{Pu}$ nucleus has been chosen as an example. One can see that the assumption of constant mass parameters, fixed at their values at the minima, is not drastically wrong. There are two reasons why a deformation dependence of mass parameters should not be crucial: 1) The relevant deformation range is limited, as the peak of the probability density of the one-phonon state corresponds to $\beta_{\lambda K}^{\pi-} \leq 0.1 ; 2$ ) Phonon 


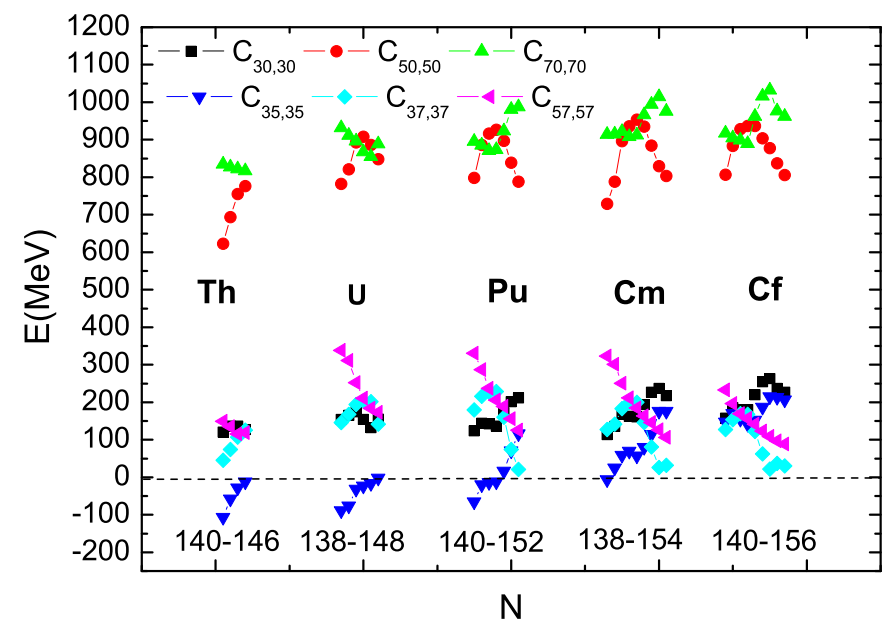

FIG. 3: Stiffness coefficients at the I-st minimum.

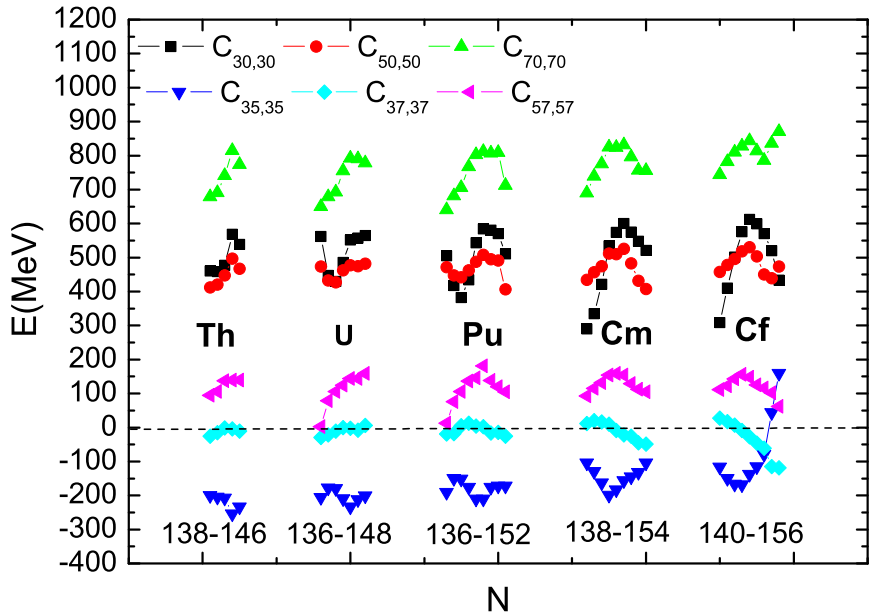

FIG. 4: Stiffness coefficients at the II-nd minimum.

energy depends on the mass parameter as $(C / B)^{1 / 2}$, which is not a very strong dependence. One can observe a different behavior of $B_{3030}$ around the I-st and II-nd minima. While at the first minimum this parameter takes the maximal (or close to the maximal) value, it reaches the minimal value at the second minimum. Both $B_{3030}$ and $B_{5050}$ are skewed with respect to the $\beta_{30}$ and $\beta_{50}$ axes.

Results of the simpler, harmonic model (3) that already contain the effect of the coupling of various multipolarities are presented below. Their modification due to the variability of the mass parameters was worked out for selected cases and is discussed afterwards. 

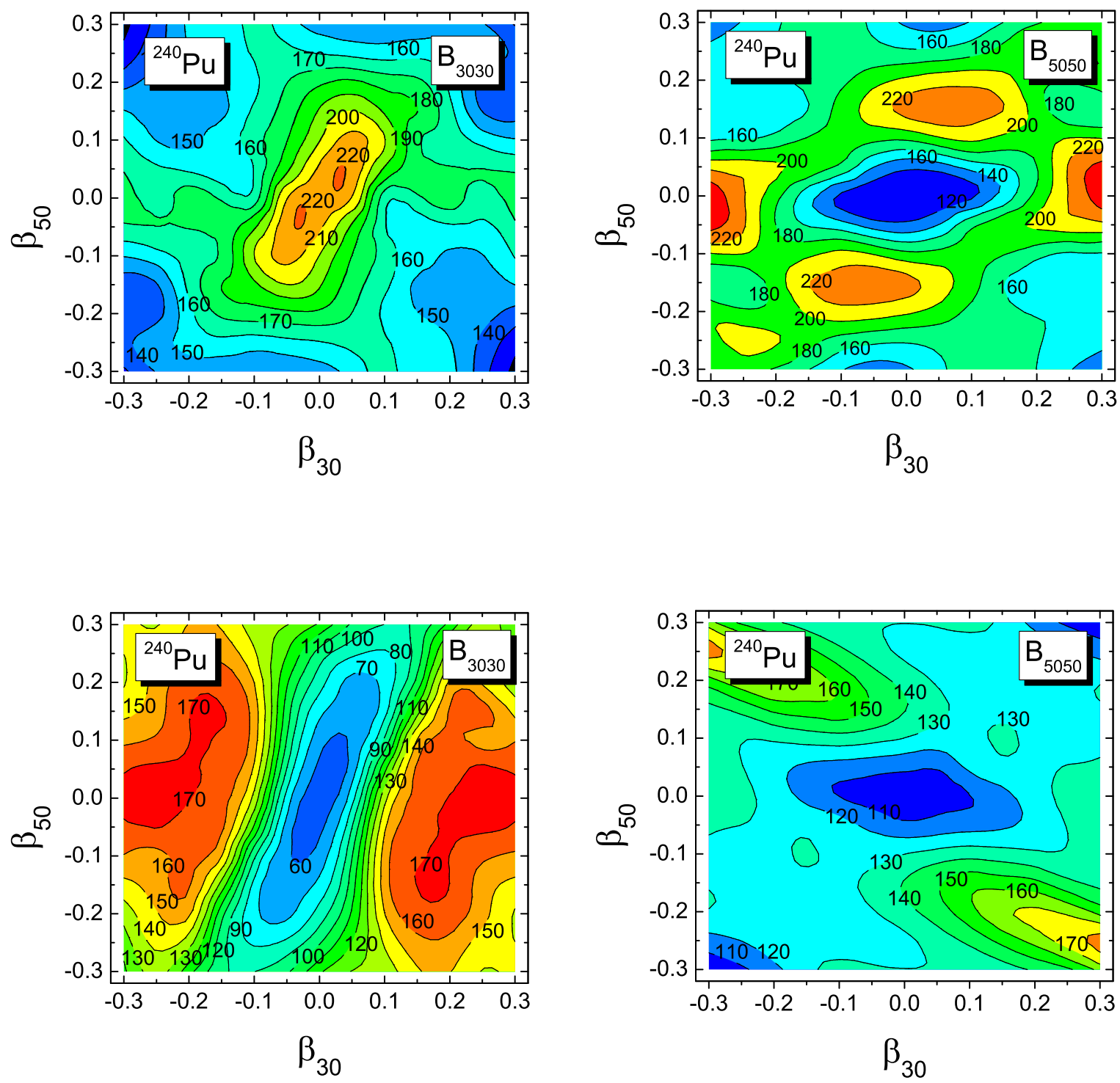

FIG. 5: Cranking mass parameters $B_{3030}, B_{5050}$ around the first (top panels) and second (bottom panels) minimum for ${ }^{240} \mathrm{Pu}$ in the $\left(\beta_{30}, \beta_{50}\right)$ plane.

\section{A. Results with constant mass parameters}

Calculated energies of the lowest negative-parity shape oscillations in the first minimum for various even-even actinides are shown in Fig. 6] together with experimental results [24]. Only vibrational cases are considered which means that we excluded nuclei with the octupole equilibrum deformation or very close to the reflection-symmetry breaking. As the calculated energies of the $K=3$ phonons are greater than $1.5 \mathrm{MeV}$ in $\mathrm{Th}, \mathrm{U}, \mathrm{Pu}$ and $\mathrm{Cm}$ isotopes and 
greater than 1.3 MeV in Cf isotopes, and, moreover, an experimental information on such octupole states is scarce and uncertain, we do not show these results here. It may be seen that for the $K=0$ and $K=1$ modes an overall agreement to within $\sim 100 \mathrm{keV}$ (except for $\left.{ }^{230} \mathrm{U}\right)$ is obtained between the data and theoretical evaluation. It is worth mentioning that our calculations do not involve any adjustment to the data they are compared with. For the $K=2$ mode the experimental energies are considerably overestimated. On the other hand, one has to notice that the experimental assignments made for $K=1$ and $K=2$ octupole band-heads are not many and some of them seem quite uncertain. One can observe in Fig. 6] a clear change in energies between $N=146$ and $N=148(150)$ : they increase for $K^{\pi}=0^{-}$ and $1^{-}$in $\mathrm{U}, \mathrm{Pu}, \mathrm{Cm}$ and $\mathrm{Cf}$, and decrease for $K^{\pi}=2^{-}$in $\mathrm{U}, \mathrm{Pu}$ and $\mathrm{Cm}$.

From the difference between the energies and the quantities $\left(C_{3 K 3 K} / B_{3 K 3 K}\right)^{1 / 2}$ one can judge the importance of the off-diagonal components of the stiffness and mass tensors. The resulting mixed multipolarity character of the lowest phonon may be also seen from the deformation parameters $\beta_{\lambda K}^{t r}$ that follow from Eq.(41). For the $K=0$ mode, they show a small admixture of $\beta_{50}$ and/or $\beta_{70}$ deformation to $\beta_{30}: \lambda=5$ mixing is important for the lightest/heaviest isotopes, $\lambda=7$ for the middle. For example, we have $\left(\beta_{30}^{t r}, \beta_{50}^{t r}, \beta_{70}^{t r}\right)=$ $(0.049,0.013,-0.015)$ in ${ }^{232} \mathrm{U},(0.058,0.005,-0.017)$ in ${ }^{238} \mathrm{U}$ and similar values in ${ }^{240} \mathrm{Pu}$ and ${ }^{246} \mathrm{Cm}$, while the $\beta_{30}-\beta_{50}$ coupling is implied by the values $(0.052,-0.017,0)$ in ${ }^{250} \mathrm{Cf}$. For the $K=1$ mode, the admixture of $\beta_{51}$ is, except for Th isotopes, larger than that of $\beta_{71}$ : in ${ }^{238} \mathrm{U}$ we have $\beta_{\lambda 1}^{t r}=(0.050,-0.016,0.007)$; similar values occur for ${ }^{240} \mathrm{Pu}$ and many other nuclei. As shown in Fig. 6, the role of the coupling to higher multipolarities decreases with the increasing $K$-number in the first potential well. In particular, for $K^{\pi}=2^{-}$vibrations, one could use the simple formula $\hbar \omega_{2}=\sqrt{\frac{C_{3232}}{B_{3232}}}$ for the excitation energy. Appreciable admixtures, mainly of $\lambda=5$, are present for the lighter isotopes.

In Fig. 7, calculated low-lying negative-parity excitation energies at the second minimum are shown as a function of the neutron number for different isotopes. The plotted experimental data were taken from [1]. The $K=3$ energies are not presented as they lie above $2.5 \mathrm{MeV}$, even higher than in the first minima. We do show calculated vibration energies in the second well in Cf isotopes, although no shape isomer was established there up to now. In our calculations, the barrier protecting the second minimum in ${ }^{246} \mathrm{Cf}$ against fission is by $1 \mathrm{MeV}$ smaller and thinner than in its isotone ${ }^{244} \mathrm{Cm}$, which supports the short-lived $(<5 \mathrm{ps})$ shape isomer and its longer lived $(<100 \mathrm{~ns})$, probably spin-isomeric, $1.3 \mathrm{MeV}$ 
excitation [25]. However, it is not completely excluded that, with a better technique, some shape isomer in Cf could be established in the future, at least in an odd isotope.

Rather extensive experimental results were collected on the excitations in the II minimum of ${ }^{240} \mathrm{Pu}[26$, 27]. These include negative-parity rotational bands as well as the candidate for the $\beta$-vibration band. The lowest rotational bands interpreted as $K^{\pi}=0^{-}, 1^{-}$and $2^{-}$have bandheads at the excitation energy $555\left(I^{\pi}=1^{-}\right), 836\left(I^{\pi}=1^{-}\right)$and $806 \mathrm{keV}\left(I^{\pi}=2^{-}\right)$, respectively. Only two first states, $I^{\pi}=1^{-}$and $3^{-}$, of the supposed octupole $K^{\pi}=0^{-}$band were observed. There are three more bands identified as $K^{\pi}=1^{-}$: at 936, 1246 and 1344 $\mathrm{keV}$; the supposed $\beta$ band starts with the second $I^{\pi}=0^{+}$state at $770 \mathrm{keV}$.

As follows form Fig,7, within the presented model we cannot reproduce the $K=0^{-}$ excitations at the second minimum. In ${ }^{240} \mathrm{Pu}$, we obtain energy nearly three times too large. Situation is even worse for ${ }^{236} \mathrm{U}$, in which the back-decay to the first minimum was experimentally detected and the excitation energy of mere $290 \mathrm{keV}$ was attributed to the $K^{\pi}=0^{-}$state in the second well. One reason for our results can be seen in Figs. 3 and 4, by comparing the stiffness $C_{3030}$ against the octupole deformation $\beta_{30}$ at the first and second minima. For all studied nuclei, the stiffness in the second minimum is roughly two times larger than in the first one and mostly larger than the stiffnes $C_{5050}$ against $\beta_{50}$. The second reason for the larger $K=0$ excitation energies in the second well comes from the mass parameters: $B_{3030}$ are two to three times smaller at the second minimum than at the g.s., and even little smaller than $B_{5050}$, opposite to the situation at the first minimum, see Fig. 5.

As a result, the lowest $K^{\pi}=0^{-}$excitation in the second well is a mixture of the $\beta_{30}$ and $\beta_{50}$ modes, with the latter dominating for nearly all isotopes. For example, the values of $\beta_{\lambda 0}^{t r}$ are equal to $(0.033,0.040,0)$ in ${ }^{230} \mathrm{Th},(0.032,0.041,-0.005)$ in ${ }^{236} \mathrm{U},(0.030,0.040,-0.007)$ in ${ }^{240} \mathrm{Pu},(0.024,0.041,0)$ in ${ }^{248} \mathrm{Cm}$ and $(0.020,0.040,0.003)$ in ${ }^{250} \mathrm{Cf}$. When the coupling between different multipolarities is neglected, the $\beta_{50}$ mode is the lowest in the second minimum.

There is a large energy lowering below the uncoupled value $\left(C_{3030} / B_{3030}\right)^{1 / 2}$ due to this coupling, see Fig. 7. This shows that it is impossible to describe low-lying $K^{\pi}=0^{-}$states in the second well as a pure octupole vibration. However, the coupling with the $Y_{50}$ mode is not sufficient in view of the experimental results.

The couplings for the $K^{\pi}=1^{-}$and $2^{-}$modes reduce phonon energies by $\sim 300$ and 
$\sim 400 \mathrm{keV}$, respectively, below the values of $\left(C_{3 K 3 K} / B_{3 K 3 K}\right)^{1 / 2}$, Fig. 7. This is much less than for the $0^{-}$mode. The values $\beta_{\lambda K}^{t r}$ show a conspicuous mixing of $\lambda=3$ and 5 , with much smaller admixtures of $\lambda=7$. For $K^{\pi}=1^{-}$phonons, the octupole mode always dominates, $\beta_{31}^{t r}>\beta_{51}^{t r}$. In most cases this is also true for the $K^{\pi}=2^{-}$mode, but for some nuclei $\beta_{52}^{t r}$ reaches the size of $\beta_{32}^{t r}$. Both phonon energies in ${ }^{240} \mathrm{Pu}$ are overestimated, by $400\left(1^{-}\right)$and $170 \mathrm{keV}\left(2^{-}\right)$; the supposed $2^{-}$band head at $830 \mathrm{keV}$ in ${ }^{236} \mathrm{U}$ is also calculated too high by $430 \mathrm{keV}$. The difference in the calculated $K^{\pi}=2^{-}$phonon energies in ${ }^{236} \mathrm{U}$ and ${ }^{240} \mathrm{Pu}$ follows mainly from the larger mass parameter $B_{3232}$ in the latter. It is remarkable that in the second minimum the mass parameters $B_{3131}>B_{3232}$ are $\sim 2$ or more times larger than $B_{3030}$, while the latter are the largest in the first minimum.

\section{B. Results with shape-dependent mass parameters}

Oscillation energies $\hbar \omega_{K}$ in the first and second minima calculated with shape-dependent mass parameters for selected even-even actinides are compared in Table \ to the constantmass variant of the calculations and the experimental data, where available. As may be seen, the model including the shape dependence of the mass parameters (2) spoils or improves the agreement with the data, depending on a particular case. In the first well, there is a substantial decrease in some $K^{\pi}=0^{-}$energies away from the measured values, especially for ${ }^{240} \mathrm{Pu}$. Some other energies become closer to the data, e.g. in ${ }^{236} \mathrm{U}$ and ${ }^{246} \mathrm{Cm}$. The $K^{\pi}=1^{-}$ energy in ${ }^{230} \mathrm{Th}$ is spoiled, while for the $K^{\pi}=2^{-}$mode there is an improvement for ${ }^{240} \mathrm{Pu}$ and worsening for ${ }^{250} \mathrm{Cf}$, already $400 \mathrm{keV}$ off the data in the harmonic approximation. These changes follow from the differences between the mass parameters at the minimum and their averages over a region around it.

In the second minimum, $K^{\pi}=0^{-}$energies from the full Hamiltonian (2) are by 200-300 $\mathrm{keV}$ lower than for its simplified version (3), except for ${ }^{234} \mathrm{U}$, for which $\hbar \omega_{0}$ stays the same. This is an improvement, but not a sufficient one, as now the $K^{\pi}=0^{-}$energy for ${ }^{240} \mathrm{Pu}$ is roughly 2.4 times too large instead of three. The $K^{\pi}=1^{-}$and $2^{-}$energies in ${ }^{236} \mathrm{U}$ and ${ }^{240} \mathrm{Pu}$ do not change much, except for the $K^{\pi}=2^{-}$state in ${ }^{240} \mathrm{Pu}$, that increases further $200 \mathrm{keV}$ away from the experimental value.

The structure of vibration phonons changes mainly according to the energy change. The values of $\beta_{\lambda K}^{t r}$ increase appreciably with respect to the constant mass parameters version only 
TABLE I: Energies of negative-parity shape vibrations (in $\mathrm{keV}$ ) for $K=0,1,2$ around I and II minimum obtained from the diagonalization of the full Hamiltonian (2) $-H_{\text {coll }}$, using constant mass parameters in the minimum (3) - $B_{\text {const }}$ and calculated by a simple formula: $\hbar \omega_{K}=\sqrt{\frac{C_{3 K 3 K}}{B_{3 K} 3 K}}$. Experimental data are taken from [24] for the I-st minima and from [1] for the II-nd minima.

\begin{tabular}{|c|c|c|c|c|c|c|c|c|c|}
\hline \multirow{3}{*}{$Z$} & \multirow{3}{*}{$A$} & & & I MIN & & & & II MIN & \\
\hline & & $H_{\text {coll }}$ & $B_{\text {const }}$ & $\sqrt{\frac{C_{3 K 3 K}}{B_{3 K 3 K}}}$ & exp & $H_{\text {coll }}$ & $B_{\text {const }}$ & $\sqrt{\frac{C_{3 K 3 K}}{B_{3 K 3 K}}}$ & $\exp$ \\
\hline & & \multicolumn{4}{|l|}{$K=0$} & \multicolumn{4}{|l|}{$K=0$} \\
\hline 92 & 230 & 551 & 527 & 756 & 367 & 1138 & 1309 & 2482 & - \\
\hline 92 & 232 & 698 & 546 & 773 & 563 & 1132 & 1331 & 2430 & - \\
\hline 92 & 234 & 820 & 689 & 906 & 786 & 1400 & 1390 & 2672 & - \\
\hline 92 & 236 & 694 & 644 & 881 & 688 & 1199 & 1430 & 3002 & 290 \\
\hline 92 & 238 & 451 & 561 & 795 & 680 & 1236 & 1492 & 3104 & - \\
\hline 94 & 238 & 465 & 529 & 814 & 605 & 1197 & 1505 & 2999 & - \\
\hline 94 & 240 & 338 & 509 & 792 & 597 & 1272 & 1565 & 3254 & 555 \\
\hline 94 & 244 & 1062 & 1083 & 1129 & $950(?)$ & 1353 & 1654 & 3339 & - \\
\hline \multirow[t]{2}{*}{96} & 246 & 1092 & 1138 & 1217 & 1079 & 1380 & 1681 & 3440 & - \\
\hline & & \multicolumn{4}{|l|}{$K=1$} & \multicolumn{4}{|l|}{$K=1$} \\
\hline 90 & 230 & 770 & 915 & 1033 & 952 & - & 962 & 1373 & - \\
\hline 92 & 236 & - & 1036 & 1175 & $967(?)$ & 1354 & 1461 & 1765 & - \\
\hline \multirow[t]{2}{*}{94} & 240 & 858 & 859 & 1015 & $938(?)$ & 1266 & 1289 & 1568 & 836 \\
\hline & & \multicolumn{4}{|l|}{$K=2$} & \multicolumn{4}{|l|}{$K=2$} \\
\hline 90 & 230 & 1264 & 1253 & 1376 & $1079(?)$ & - & 1367 & 1760 & - \\
\hline 92 & 234 & 1377 & 1348 & 1488 & 989 & - & 1287 & 1704 & - \\
\hline 92 & 236 & - & 1385 & 1490 & $1110(?)$ & 1345 & 1226 & 1639 & $830(?)$ \\
\hline 94 & 240 & 1295 & 1469 & 1497 & $1241(?)$ & 1166 & 972 & 1355 & 806 \\
\hline 98 & 250 & 1303 & 1274 & 1277 & 872 & - & 1061 & 1738 & - \\
\hline
\end{tabular}

if there is an appreciable decrease in the phonon energy. Their ratios are similar as those for the harmonic Hamiltonian. It should be noticed that for anharmonic vibrations the simple relation between $\beta_{\lambda K}^{t r}$ and the multipolarity composition of the vibration coordinate, based on Eq.(4), is lost.

One can say that the results obtained by the full diagonalization of the Hamiltonian (2) do not introduce drastic changes in the results obtained with (3).

\section{Electromagnetic dipole transitions}

Transition dipole moments governing E1 transitions between one-phonon $K=0$ and $K=1$ bands and the g.s. band have been calculated using the values $\beta_{\lambda K}^{t r}$ from the model 
(3) with constatnt mass parameters. They are given in Table II for selected nuclei. At the first minimum, $D^{t}$ values show a considerable variation from a nucleus to nucleus. The $K=0$ and 1 components are correlated, e.g. both are large for ${ }^{230} \mathrm{U}$; this large $K=0$ value is roughly consistent with the measurements [28]. For $K=1, B(E 1) \sim 2\left(D^{t}\right)^{2}$, Eq. (5) , so for an easy comparison of $B(E 1)$ s for $K=0$ and $K=1$, one should multiply $D^{t}$ for $K=1$ from Table II by $\sqrt{2}$. For some nuclei, the opposite signs of the microscopic and macroscopic parts lead to a nearly complete cancellation of $D^{t}$. This results in a variation of the deexcitation pattern along the isotopic chain, as may be seen for ${ }^{230-238} \mathrm{U}$.

More specifically, the negative-parity rotational band decays according to the ratios of the rates of E2 transitions within, and E1 transitions out of the band. From the calculated transition dipole moments $D^{t}$ in $[e \mathrm{fm}]$ we can estimate the ratio

$$
\frac{T(E 1)_{I \rightarrow I-1}}{T(E 2)_{I \rightarrow I-2}}=1.303 \frac{E_{\gamma}^{3}(E 1)}{E_{\gamma}^{5}(E 2)}\left(\frac{8}{5}\right) \frac{(2 I-1)(I-1)}{(I-1)^{2}-K^{2}}\left(\frac{D^{t}}{Q_{0}}\right)^{2},
$$

where $Q_{0}$ is the intrinsic quadrupole moment of the negative-parity band in units of [10 b], and energies $E_{\gamma}$ of gamma transitions are given in $\mathrm{MeV}$. Using values of $Q_{0}$ measured for the g.s. bands, we obtain the following $\mathrm{T}(\mathrm{E} 1) / \mathrm{T}(\mathrm{E} 2)$ ratios for transitions from the $I^{\pi}=7^{-}$ state of the $K^{\pi}=0^{-}$band: around 300 in ${ }^{232,234} \mathrm{U}, 1.6$ in ${ }^{236} \mathrm{U}$ and around 100 in ${ }^{238} \mathrm{U}$. Although not a perfect match with tha data, this roughly correlates with no intraband E2s seen along the negative-parity bands in ${ }^{232,234} \mathrm{U}$, the complete regular E2 cascade and no E1s above the $3^{-}$state in ${ }^{236} \mathrm{U}$, and the $\mathrm{E} 2$ cascade ending at the $7^{-}$state, with more E1s in ${ }^{238} \mathrm{U}$ [29 31]. On the other hand, the predicted nearly perfect cancellation of $D^{t}$ for $K=0$ in ${ }^{240} \mathrm{Pu}$ is not supported by the value $\approx 0.12$ efm measured at spin 11 and the smaller values for the neighbouring $\mathrm{Pu}$ isotopes [32]. The relatively small dipole moment measured for ${ }^{230} \mathrm{Th}$ [33] does not contradict our $K=1$ value.

In the isomeric second minimum the situation is entirely different. The macroscopic part, $D_{\text {mac }} \sim \beta_{20} \beta_{3 K}$ in the leading order, is dominant there as its magnitude becomes larger than that of the shell-correction part due to the large equilibrium value of $\beta_{20}$. For $K=0$ bands, the microscopic part adds to the macroscopic part. The large values of the dipole moment and small energies of rotational transitions near the bandheads preclude observation of the intraband E2s in negative-parity vibrational bands in the isomeric minima in actinides. This agrees with the lack of experimentally observed E2 transitions in the isomeric minimum in ${ }^{240} \mathrm{Pu}$ [1, 26, 27]. 
TABLE II: Macroscopic $D_{m a c}$, microscopic $D_{m i c}$ and total transition dipole moment $D^{t}$ for $K^{\pi}=$ $0^{-}$and $1^{-}$phonons in the first and second minimum.

\begin{tabular}{|c|c|c|c|c|c|c|c|}
\hline \multirow[b]{2}{*}{$Z$} & \multirow[b]{2}{*}{$A$} & \multicolumn{3}{|c|}{ I MIN } & \multicolumn{3}{|c|}{ II MIN } \\
\hline & & $D_{m a c}$ & $D_{m i c}$ & $D^{t}$ & $D_{m a c}$ & $D_{m i c}$ & $D^{t}$ \\
\hline & & $K=0$ & & & $K=0$ & & \\
\hline 92 & 230 & 0.19 & 0.04 & 0.23 & 0.29 & 0.11 & 0.40 \\
\hline 92 & 232 & 0.17 & 0.00 & 0.17 & 0.34 & 0.08 & 0.42 \\
\hline 92 & 234 & 0.17 & -0.07 & 0.10 & 0.34 & 0.04 & 0.38 \\
\hline 92 & 236 & 0.17 & -0.16 & 0.01 & 0.34 & 0.02 & 0.35 \\
\hline 92 & 238 & 0.17 & -0.24 & -0.07 & 0.33 & 0.02 & 0.35 \\
\hline 94 & 238 & 0.19 & -0.13 & 0.06 & 0.33 & 0.09 & 0.42 \\
\hline 94 & 240 & 0.18 & -0.18 & 0.00 & 0.32 & 0.08 & 0.40 \\
\hline 94 & 244 & 0.15 & -0.18 & -0.03 & 0.29 & 0.03 & 0.32 \\
\hline \multirow[t]{2}{*}{96} & 246 & 0.15 & -0.14 & 0.01 & 0.29 & 0.12 & 0.41 \\
\hline & & $K=1$ & & & $K=1$ & & \\
\hline 90 & 230 & -0.042 & 0.031 & -0.01 & -0.157 & -0.077 & -0.23 \\
\hline 92 & 230 & -0.049 & -0.079 & -0.13 & -0.173 & -0.055 & -0.23 \\
\hline 92 & 232 & -0.047 & -0.041 & -0.09 & -0.179 & -0.035 & -0.21 \\
\hline 92 & 234 & -0.049 & 0.008 & -0.04 & -0.174 & -0.017 & -0.19 \\
\hline 92 & 236 & -0.055 & 0.035 & -0.02 & -0.170 & 0.00 & -0.17 \\
\hline 92 & 238 & -0.063 & 0.054 & -0.01 & -0.174 & 0.00 & -0.17 \\
\hline 94 & 238 & -0.059 & 0.024 & -0.036 & -0.182 & 0.023 & -0.16 \\
\hline 94 & 240 & -0.065 & 0.045 & -0.02 & -0.183 & 0.022 & -0.16 \\
\hline
\end{tabular}

\section{CONCLUSIONS}

Our study of low-lying negative-parity oscillations in even-even actinides leads to the following conclusions:

- Considering that we have no adjustable parameters, the data on negative-parity excitations in the first minima are reproduced quite well for the $K^{\pi}=0^{-}$and $1^{-}$phonons; 
energies of the $2^{-}$phonons are significantly overestimated.

- The model predicts $K^{\pi}=0^{-}$energies in the second well in ${ }^{240} \mathrm{Pu}$ and ${ }^{236} \mathrm{U}$ that are three and more times larger than the claimed experimental values. For the $K=1,2$ phonons, the agreement with the data is better, similar to that obtained in [11] for ${ }^{240} \mathrm{Pu}$, but still the calculated energies are too high.

- Low-lying negative-parity oscillations show small admixtures of the multipolarities $\lambda=$ 5,7 to the octupole mode at the first minima, and equal or dominant contribution of the $\lambda=5$ multipole at the second minimum. Hence the coupling of various multipolarities is important in the description of the "octupole" vibrations, especially in the second minimum.

- Taking into account the multipoles $\lambda=3,5,7$ in the phonon structure and the exact macroscopic contribution to the dipole moment, we predict large transition dipole moments from the "octupole" band to the g.s. band in lighter actinides at the first minima, and for all investigated nuclei at the isomeric minima.

We do not see any way to reconcile our model with the reported data on the energies of the $K^{\pi}=0^{-}$mode in the second well. Both the calculated sizable stiffness Fig. 4 and small mass parameters Fig. 5 suggest that either our model is completely unreliable there, or, perhaps, the experimental $K^{\pi}=0^{-}$assignments in the shape isomeric minima in ${ }^{236} \mathrm{U}$ and ${ }^{240} \mathrm{Pu}$ are not related to the collective shape vibrations.

One could think of possible improvements of the model. A natural step would be to include the quadrupole-octupole coupling. However, as long as the second minima are reflection-symmetric, this would be a second-order effect, while the energy surfaces and mass parameters do not hint to its unusual enhancement. One could also consider a finetuning of the pairing strength in the second well, or including the quadrupole pairing as in [11]. Still, the required reduction of the $0^{-}$phonon energies is so large, that the pairing alone hardly can be a cause. On the other hand, the discrepancies observed for $1^{-}$and $2^{-}$phonons in the II-nd well probably could be reduced by a fine tuning of the model parameters. 


\section{Appendix A: Some aspects of the calculations}

The matrix diagonalization of the Hamiltonian (2) is performed in the basis of the threedimensional harmonic oscillator. The matrix elements of the Hamiltonian were calculated with the 20 point Gauss-Hermitte quadratures. The calculations were reduced by setting the mass parameters to their values at the minimum outside the mesh of $14 \times 14 \times 14$ points for the easier $K=0$ case, and outside the mesh of $8 \times 8 \times 6$ points for $K=1$ and 2 . We have checked that the related distortion of the Hamiltonian is unimportant for the lowest phonon states, as even the smaller mesh covers their peak region. As a check of the program, we reproduced the harmonic case of constant mass parameters. We also checked the case of the position-dependent mass parameter $B \sim \beta^{2}$ with the quartic potential $\sim \beta^{4}$, which gives the harmonic spectrum.

The cranking mass parameters were calculated by replacing derivatives with respect to deformations by finite differences. This has some advantages for many deformation parameters, but introduces an error which may reach $2-3 \%$ for diagonal components. The new approach was tested with the old code for mass parameters for axial and some nonaxial deformations. The main tool of these calculations, the code that diagonalizes the s.p. Hamiltonian, was checked independently with the older, less general versions.

An independent test of this code is provided by the fact, that different deformation sets may correspond to the same shape, so they should produce the same s.p. spectrum. In particular, the deformations $Y_{\lambda K s}$ with $K=1,3, \lambda=3,5,7$ of an axially symmetric equilibrium shape give the same s.p. spectrum as the deformations $Y_{\lambda K c}$. The latter are accomodated by the parametrization Eq. (1), by switching the choice of the symmetry axis from $z$ to $y$. This follows from the relations between spherical harmonics defined with respect to the reference axes $z, x, y$ (denoted as $Y^{y}$ ) and the standard ones:

$$
\begin{aligned}
Y_{31 c}^{y} & =\sqrt{\frac{3}{8}} Y_{30}+\sqrt{\frac{5}{8}} Y_{32 c} \\
Y_{33 c}^{y} & =-\sqrt{\frac{5}{8}} Y_{30}+\sqrt{\frac{3}{8}} Y_{32 c} \\
Y_{51 c}^{y} & =-\sqrt{\frac{15}{64}} Y_{50}-\sqrt{\frac{7}{16}} Y_{52 c}-\sqrt{\frac{21}{64}} Y_{54 c} \\
Y_{53 c}^{y} & =\sqrt{\frac{35}{128}} Y_{50}+\sqrt{\frac{3}{32}} Y_{52 c}-\frac{9}{\sqrt{128}} Y_{54 c}
\end{aligned}
$$




$$
\begin{aligned}
Y_{71 c}^{y} & =\frac{5 \sqrt{7}}{32} Y_{70}+\frac{15 \sqrt{7}}{32 \sqrt{2}} Y_{72 c}+\frac{3 \sqrt{33}}{32} Y_{74 c}+\frac{\sqrt{429}}{32 \sqrt{2}} Y_{76 c}, \\
Y_{73 c}^{y} & =-\frac{3 \sqrt{21}}{32} Y_{70}-\frac{19}{32 \sqrt{2}} Y_{72 c}+\frac{\sqrt{11}}{32} Y_{74 c}+\frac{3 \sqrt{143}}{32 \sqrt{2}} Y_{76 c} .
\end{aligned}
$$

The equivalent deformation set for axially symmetric first and second minima follows from the expressions for spherical harmonics $Y_{\lambda 0}^{y}$ :

$$
\begin{aligned}
& Y_{40}^{y}=\frac{3}{8} Y_{40}+\frac{\sqrt{5}}{4} Y_{42 c}+\frac{\sqrt{35}}{8} Y_{44 c}, \\
& Y_{60}^{y}=-\frac{5}{16} Y_{60}-\frac{\sqrt{105}}{16 \sqrt{2}} Y_{62 c}-\frac{3 \sqrt{7}}{16} Y_{64 c}-\frac{\sqrt{231}}{16 \sqrt{2}} Y_{66 c}, \\
& Y_{80}^{y}=\frac{35}{128} Y_{80}+\frac{3 \sqrt{35}}{32 \sqrt{2}} Y_{82 c}+\frac{3 \sqrt{77}}{64} Y_{84 c}+\frac{429}{32 \sqrt{2}} Y_{86 c}+\frac{3 \sqrt{715}}{128} Y_{88 c} .
\end{aligned}
$$

\section{Appendix B: Dipole moments within the microscopic-macroscopic method}

The macroscopic part of the expectation value of the electric diple moment is calculated as a sum of the redistribution and the neutron skin effects [21]

$$
\mathbf{D}=\mathbf{D}_{\text {red }}+\mathbf{D}_{\text {skin }},
$$

where for $Z$ protons and $N$ neutrons, $A=Z+N, I=(N-Z) / A$, one obtains from the Droplet Model

$$
\begin{aligned}
\mathbf{D}_{\text {red }}= & \frac{A Z e^{2}}{8}\left(\frac{1}{J}+\frac{6 L I}{K J}\right)\left(<v>_{V}<\xi>_{V}-<v \xi>_{V}\right), \\
\mathbf{D}_{\text {skin }} & =\frac{2 N Z}{A}(I-\bar{\delta}) R_{0}\left(<\xi>_{V}-<\xi>_{S}\right) \\
& +\frac{9}{32} \frac{Z A^{2 / 3} e^{2}}{Q} B_{S}\left(<v>_{S}<\xi>_{S}-<v \xi>_{S}\right) .
\end{aligned}
$$

The Droplet Model parameters are: the volume symmetry-energy coefficient $J$, the nuclear incompressibility $K$, the effective neutron skin stiffness $Q$, the density symmetry coefficient $L$, the nuclear radius $R_{0}=r_{0} A^{1 / 3}$ and the equilibrium value of the average relative neutron excess,

$$
\bar{\delta}=\frac{I+\frac{9 e^{2}}{80_{0} Q} Z A^{-2 / 3}}{1+\frac{9 J}{4 Q} A^{-1 / 3}} .
$$


Nuclear shape enters through the constant $B_{S}$, being the ratio of the area of a deformed surface to the surface area of the sphere of the same volume, and the averages, over both the nuclear volume and surface, of the scaled radius vector $\xi=\mathbf{r} / R_{0}$ and the Coulomb potential $v$ in units of $Z e / R_{0}$, with $<f>_{V}$ meaning $\int_{V} f / V$, and $\left\langle f>_{S}\right.$ meaning $\int_{S} f / S$. The calculations were performed with the following values of the parameters: $r_{0}=1.16 \mathrm{fm}$, $J=32.5 \mathrm{MeV}, K=240 \mathrm{MeV}, Q=50 \mathrm{MeV}$ and $L=100 \mathrm{MeV}$, as in [22].

The microscopic part of the dipole moment is calculated as $\left.D^{\text {micr }}=\zeta(<\hat{D}\rangle-\tilde{D}\right)$ [19, 20], where the first part is the expectation value of the dipole operator on the deformed state and $\tilde{D}$ is the analogous expression in which the actual pairing occupation numbers $2 v_{i}^{2}$ were replaced by the quantities smoothed according to the Strutinsky prescription. The factor $\zeta$ takes care of the reduction of the effective charge due to the particle-vibration coupling to the $E 1$ giant resonance. We have used $\zeta=0.33$ as it was done in previous calculations [20, 22].

[1] P. G. Thirolf and D. Habs, Prog. Part. Nucl. Phys. 49, 325 (2002); P. Thirolf, D.Dc. Thesis, Ludwig-Maximilians-Universitat Munchen (2003).

[2] S. M. Polikhanov et al., Sov. Phys. JETP 15, 1016 (1962).

[3] G. N. Flerov and V. A. Druin, Preprint R-2539, JINR, Dubna (1966).

[4] V. M. Strutinsky, Nucl. Phys. A 95, 420 (1967).

[5] H. J. Specht, J. Weber, E. Konecny and D. Heunemann, Phys. Lett. B 41, 43 (1972).

[6] V. Metag, D. Habs and H. J. Speth, Phys. Rep. 65, 1 (1980).

[7] H. Backe et al., Phys. Rev. Lett. 80, 920 (1980).

[8] V. E. Makarenko, INDC(CCP)-394 (1995).

[9] K. Neergard and P. Vogel, Nucl. Phys. A 145, 33 (1970), Nucl. Phys. A 149, 217 (1970).

[10] T. Nakatsukasa, K. Matsuyanagi, K. Mizutori and Y. R. Shimizu, Phys. Rev C 53, 2213 (1996).

[11] V.G. Soloviev, A. Sushkov, and N. Yu. Shirikova, Z. Phys. A 358, 117 (1997).

[12] S. Ćwiok, J. Dudek, W. Nazarewicz, J. Skalski and T. Werner, Comput. Phys. Commun. 46, 379 (1987).

[13] H. J. Krappe, J. R. Nix and A. J. Sierk, Phys. Rev. C 20, 992 (1979). 
[14] M. Kowal, P. Jachimowicz, A. Sobiczewski, Phys. Rev. C 82, 014303 (2010).

[15] S. Ćwiok, W. Nazarewicz, J .X . Saladin, W. Płóciennik and A. Johnson, Phys. Lett. B 322, 304 (1994).

[16] J.-P. Delaroche, M. Girod, H. Goutte and J. Libert, Nucl. Phys. A 771, 103 (2006).

[17] L. M. Robledo, J. L. Egido, B. Nerlo-Pomorska and K. Pomorski, Phys. Lett. B 201, 409 (1988).

[18] A. Bohr, B. R. Mottelson, Nuclear Structure Vol. 2 (Benjamin,New York,1975)

[19] G. A. Leander, W. Nazarewicz, G. F. Bertsch and J. Dudek, Nucl. Phys. A 453, 58 (1986).

[20] P. A. Butler and W. Nazarewicz, Nucl. Phys. A 533, 249 (1991).

[21] C. O. Dorso, W. D. Myers and W. J. Swiatecki, Nucl. Phys. A 451, 189 (1986).

[22] J. Skalski, Phys. Rev. C 49, 2011 (1994).

[23] M. Schmorak et al., Nucl. Phys. A 178, 410 (1972).

[24] ,,TABLE OF ISOTOPES” edited by R. B. Firestone and V. S. Shirley, ISBN 0471-14918-7 Eight Edition, Vol 2, (1996).

[25] H. C. Britt, S. C. Burnett, B. H. Erkkila, J. E. Lynn and W. E. Stein, Phys. Rev. C 4, 1444 (1971); G. Sletten, V. Metag and E. Liukkonen, Physics Letters B 60, 2 (1976).

[26] D. Pansegrau et al, Phys. Lett. B 484, 1 (2000).

[27] D. Gassmann et al., Physics Letters B 497, 3-4, 11 (2001).

[28] B. Ackermann et al., Nucl. Phys. A 559, 61 (1993).

[29] P. Zeyen et al, Z. Phys. A 328, 399 (1987).

[30] E. Browne and J. K. Tuli, Nuclear Data Sheets 107, 2649 (2006).

[31] K. Mc Gowan and W. T. Milner, Nucl. Phys. A 571, 569 (1994); D. Ward et al., Nucl. Phys. A 600,88 (1996).

[32] I. Wiedenhöver et al., Phys. Rev. Lett. 83, 2143 (1999).

[33] Ch. Lauterbach et al., Phys. Lett. B 130, 187 (1984). 

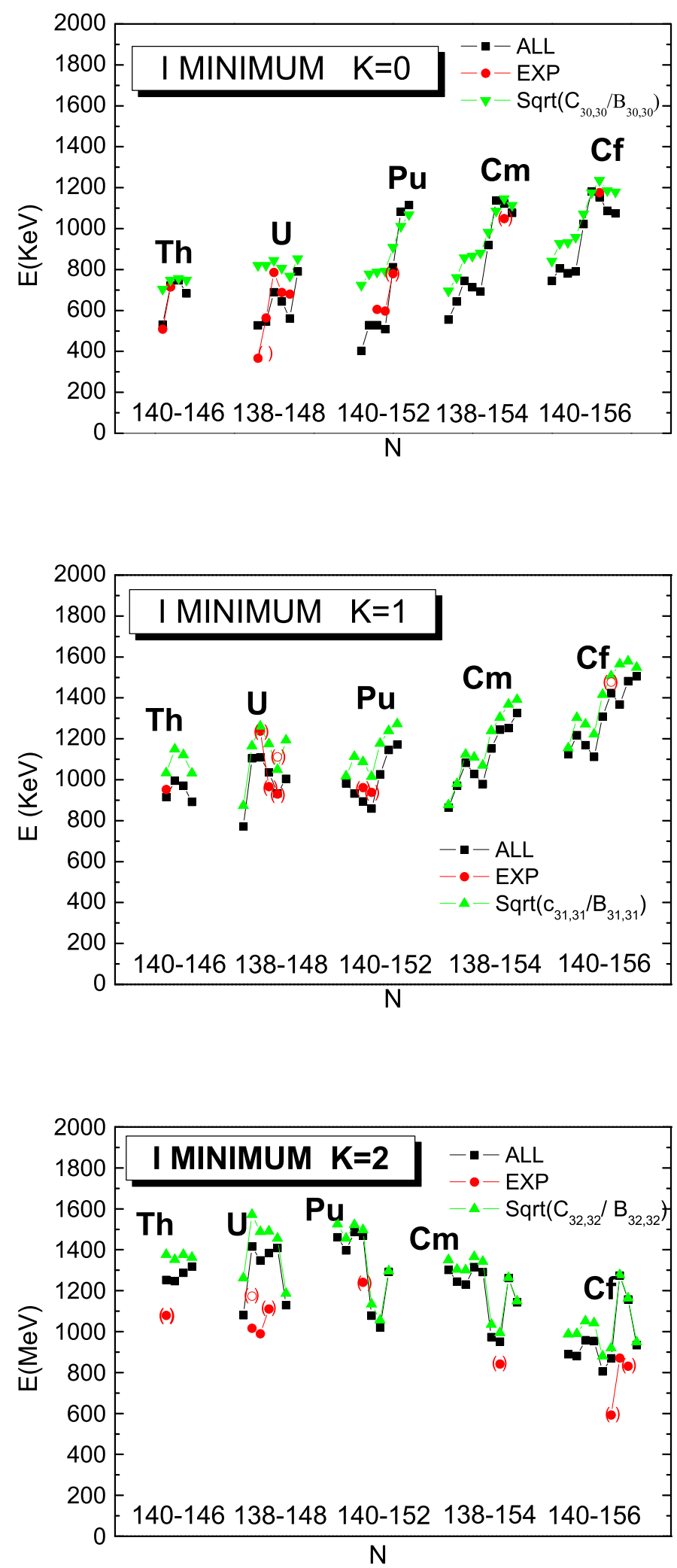

FIG. 6: Energies of negative-parity shape oscillations in the g.s. minimum for $K=0,1,2$. Results including all (ALL) multipolarities $\lambda=3,5,7$ are denoted by squares; triangles follow the formula: $\hbar \omega_{K}=\sqrt{\frac{C_{3 K 3 K}}{B_{3 K 3 K}}}$. Experimental data (EXP) [24] are marked with circles; parenthesis signals some uncertainty in the experimental assignment. 

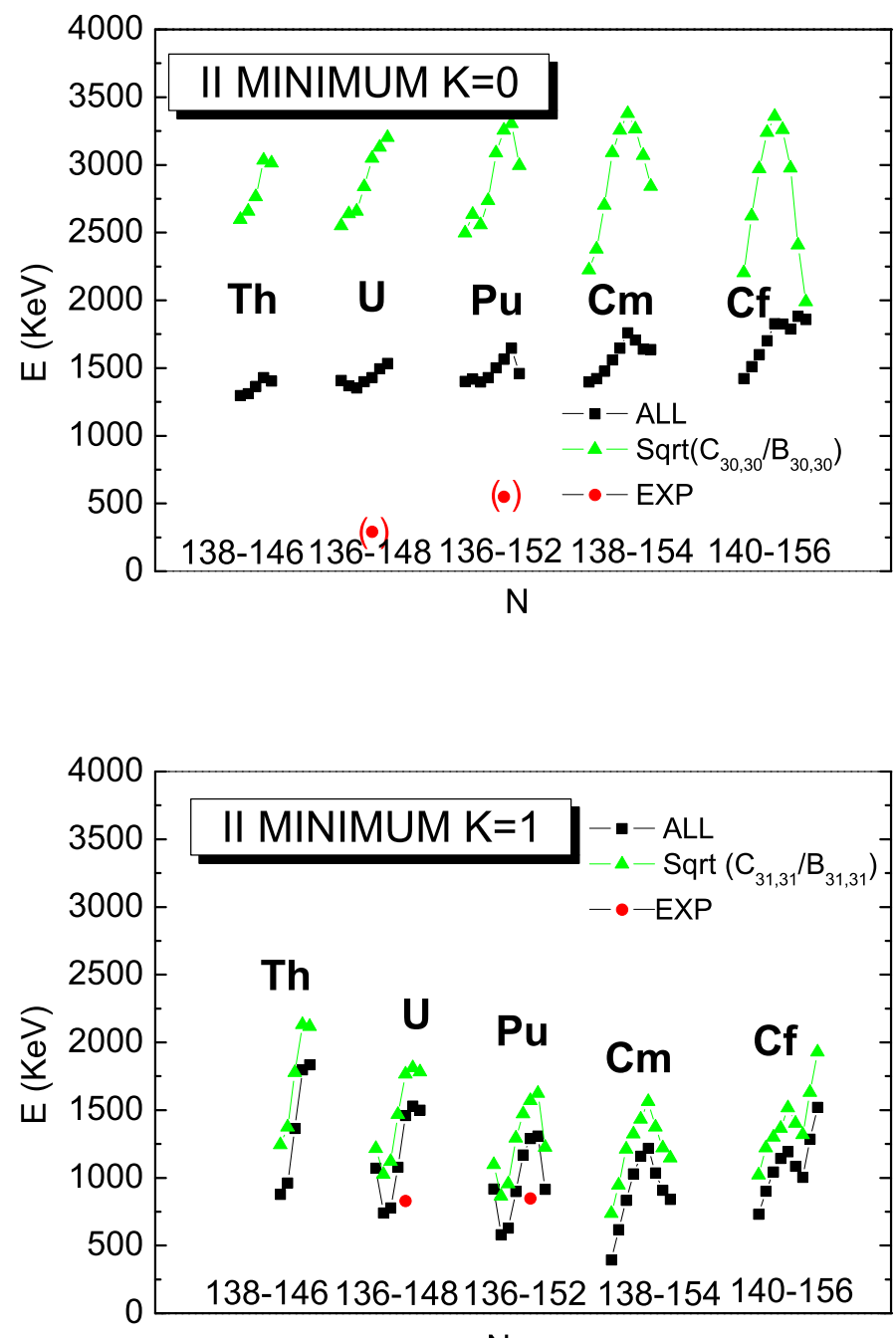

$\mathrm{N}$

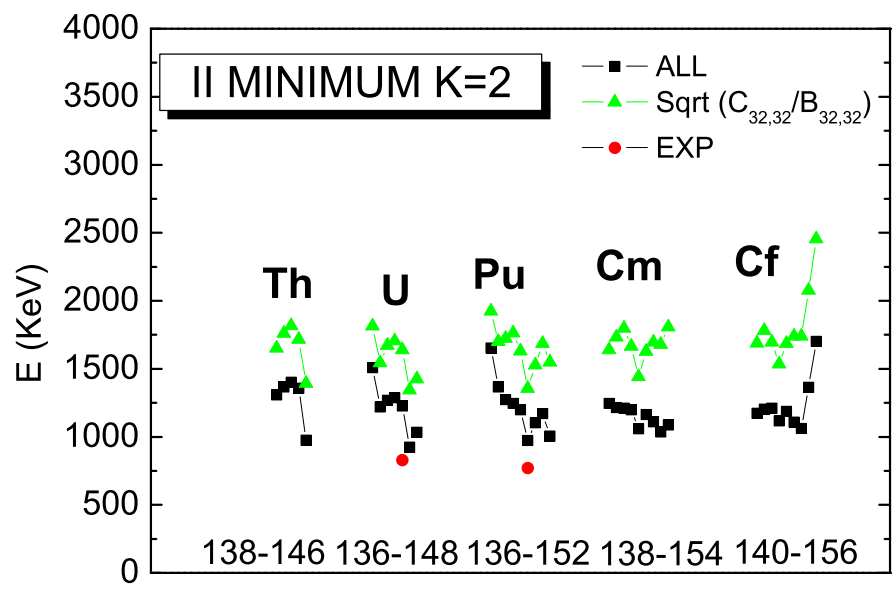

$\mathrm{N}$

FIG. 7: The same as in Fig [6, but in the second minimum. Experimental data (EXP) are taken from [1]. 